\section{Aspectos estruturais e processuais da vigilância do crescimento de menores de cinco anos em serviços públicos de saúde do Estado de Pernambuco}

\section{Process and structural aspects of monitoring growth in children under the age of five years at public health services in the state of Pernambuco, Brazil}

Cristiane de Albuquerque Silva Ratis

Departamento de Nutrição

Programa de Pós-Graduação em Nutrição

Universidade Federal de Pernambuco

Av. Prof. Moraes Rego s/n - Cidade Universitária

50670-901 Recife - PE

ratiscas@bol.com.br

\section{Malaquias Batista Filho}

Departamento de Nutrição

Programa de Pós-Graduação em Nutrição

Universidade Federal de Pernambuco

Bolsista CNPq (Processo n. 523528/95-7 (NV))

\section{Resumo}

Objetivo: Avaliar aspectos estruturais e processuais da vigilância do crescimento em 120 unidades públicas de saúde de Pernambuco. Método: O estudo foi do tipo transversal, baseado na pesquisa "Atenção à Saúde Materno-Infantil do Estado de Pernambuco”, com uma amostra de 1.669 crianças menores de cinco anos, representativa para o Estado (Região Metropolitana do Recife - RMR e Interior). O processamento e a análise dos dados foram realizados no Epi-info 6.02. Utilizou-se o qui-quadrado de Pearson, com correção de Yates, para as tabelas binárias. Resultados: Os serviços de saúde dispunham em $97,1 \%$, na RMR, de cartão para registro de dados do crescimento, proporção que cai significativamente $(80,4 \%)$ no Interior $(\mathrm{p}<0,01)$. A existência de balanças pediátricas para pesagem de menores de 2 anos foi notificada em quase $90 \%$ das unidades da RMR e em 76,5\% no Interior. Em relação à existência de normas e fichas para o acompanhamento do crescimento, as respostas positivas no Estado caíram significantemente para $23,3 \%$ e $18 \%$, respectivamente. Em $54,1 \%$, as crianças foram pesadas e $16,2 \%$ medidas (comprimento em estatura). Os acompanhantes das crianças receberam informações sobre o crescimento de suas crianças em $18,2 \%$ da amostra. Nos atendimentos, os profissionais da RMR solicitam mais o cartão que aqueles do Interior. Das crianças que apresentaram o cartão, 38\% possuíam nenhum ou apenas um ponto de registro de peso. Conclusão: Os achados indicam que, apesar dos recursos estruturais, os serviços de saúde de Pernambuco apresentamum desempenho grosseiramente deficiente no que se refere ao cumprimento de um processo efetivo e eficaz de vigilância ao crescimento de menores de cinco anos.

Palavras-chave: Avaliação dos serviços. Crescimento. Vigilância nutricional. 


\section{Abstract}

Objective: To evaluate the process and structural aspects of growth monitoring in 120 public health units in the state of Pernambuco in Northeast Brazil. Method: We carried out a cross-sectional study based on a research survey entitled "Child and Maternal Health Care in the State of Pernambuco". The study included 1,669 children under the age of five years representing both Metropolitan Recife (state capital) and the Interior Regions of the State. Data processing and analysis were performed using Epi-info 6.02 software. The Pearson chisquared test with the Yates correction was used for the binary tables. Results: Health services in $97.1 \%$ of Metropolitan Recife (MR) have record cards for growth data available. This proportion drops considerably (80.4\%) in the Interior Regions $(\mathrm{p}<0.01)$. Pediatric scales for weighing children under the age of 2 years was reported in nearly $90.0 \%$ of MR units, and just $76.5 \%$ in those in the Interior Regions. Regarding standards and charts for monitoring growth, positive responses dropped significantly in the different regions of the state $-23.3 \%$ and $18.0 \%$, respectively. In $54.1 \%$ of the cases, children were weighed and in $16.2 \%$ growth measures were taken (height length). Parents or guardians received information on the growth of their children in $18.2 \%$ of the sample. As to health services, professionals from MR request growth cards more than those in the State Interior. 38.0\% of the children that presented the card had either no records or just one record of weight. Conclusion: The findings indicate that, despite structural resources, public health services in the State of Pernambuco present a grossly deficient performance in regard to carrying out an effective and efficient growth monitoring process for children under the age of five years.

Keywords: Services evaluation. Growth. Nutritional surveillance.

\section{Introdução}

Sob o aspecto conceitual e já agora em nível normativo, o crescimento e desenvolvimento constituem processos básicos da psicobiologia da criança. São instâncias fundamentais de diferenciação para a vida adulta, constituindo, na atenção à saúde, os eixos referenciais dos cuidados prestados às crianças, acompanhando ou seguindo-se à preocupação imediatista de se contornar os riscos mais visíveis relacionados à própria sobrevivência, nos primeiros meses de vida ${ }^{1}$.

O reconhecimento científico, técnico e programático da vigilância do processo de crescimento e desenvolvimento da criança difundiu-se há pouco mais de meio século, com a emergência simultânea da chamada pediatria social com Debret na França, Richmond nos Estados Unidos, Morley na Inglaterra, Frederico Gómez no México e Pedro Alcântara no Brasil ${ }^{2,3}$. Na década de 80, a experiência bem sucedida de alguns países (Chile, Cuba, Costa Rica e Jamaica, nas Américas; Siri Lanka, Filipinas e outros, na Ásia e África) propiciaram o pretexto para a internacionalização das ações básicas e integradas na atenção à saúde e nutrição das crianças ${ }^{4}$.

A experiência destes países na aplicação extensiva das ações básicas de saúde, incluindo a monitoração normativa do processo de crescimento e desenvolvimento, serviu de fundamento, na década de 90, para uma proposta internacional ambiciosa: o plano mundial pela sobrevivência e desenvolvimento das crianças, aprovado na reunião de Cúpula de Nova York (1990), passando a vigorar como um compromisso político e programático de 159 países, inclusive o Brasil $^{5}$.

Segundo este compromisso, os países signatários se obrigavam, até o ano 2000 , a cumprir um conjunto de metas e estratégias, entre as quais figurava a universalização do monitoramento do crescimento e desenvolvimento das crianças nos primeiros anos de vida. Na realidade, antes mesmo de assumir o pacto internacional, o Brasil já havia incluído em suas propostas de atenção à saúde da criança a incorporação da vigilância 
do crescimento e desenvolvimento como uma diretriz básica, mediante a institucionalização do Programa de Assistência Integral à Saúde da Criança (PAISC, 1984), dispondo, portanto, de tempo suficiente para sua plena implantação $0^{6}$, principalmente com a ênfase que lhe foi conferida nos anos 90 .

A realização, no Estado de Pernambuco, de um estudo avaliativo da assistência materno-infantil em serviços públicos de saúde, abrangendo 120 unidades da Região Metropolitana do Recife-RMR e do Interior do Estado - IE, propiciou uma oportunidade singular de se analisar aspectos qualitativos e quantitativos da atenção prestada às crianças e às mães através das unidades básicas de saúde.

Segundo Donabedian (1985), aspectos de estrutura e processo são fundamentais para se compreender a instância ou objetivo final da própria atenção: a prevenção e o controle de situações mórbidas ou disbiógenas ${ }^{7}$. No caso da monitoração do crescimento nas condições epidemiológicas do Estado de Pernambuco e do próprio Nordeste do Brasil, a utilidade mais imediata da monitoração do crescimento se refere à vigilância do estado nutricional das crianças, um aspecto prioritário no quadro de demandas do setor saúde ${ }^{8}$.

A partir de um banco de dados, objetivase descrever e analisar, neste artigo, aspectos referentes à monitoração do crescimento em 120 unidades de saúde do tipo I e II no Estado de Pernambuco, considerando duas vertentes: estrutura e processo.

\section{Material e Métodos}

O Estado de Pernambuco possui uma área de 98.526,6 km² e abriga uma população de 7.911.937 habitantes, dos quais 6.052.930 residem na área urbana $(76,5 \%)$, com uma densidade demográfica de 80,3 habitantes por quilômetro quadrado ${ }^{9}$. Os indicadores de saúde para o estado refletem uma situação de extrema desigualdade entre os espaços geográficos. O coeficiente de mortalidade infantil na RMR em 1998, foi de 33 por mil nascidos vivos, alcançando no
Interior rural 69 por mil. $\mathrm{O}$ baixo peso ao nascer atingiu 7,9\% no Estado como um todo. Os serviços públicos de saúde foram os mais utilizados, abrangendo $95,4 \%$ dos usuários para a área rural, $77 \%$ para a área urbana do Interior e $67,9 \%$ para a RMR. Das crianças menores de 5 anos, 4,9\% e 12,1\% apresentavam déficits moderado e grave nas relações peso/idade e estatura/idade, respectivamente $^{10,11}$.

O presente estudo, do tipo transversal, baseia-se no banco de dados da pesquisa "Atenção à Saúde Materno-Infantil no Estado de Pernambuco" realizada no período de 15 de outubro a 18 de dezembro de 1998 , com amostra representativa, distribuída em dois espaços geográficos: Região Metropolitana do Recife (RMR) e Interior do Estado ${ }^{12}$. A seleção amostral foi do tipo probabilística (aleatória estratificada) e incluiu todos os serviços pertencentes à rede pública ambulatorial de nível de hierarquia I e II. Foram incluídos no estudo os seguintes elementos amostrais: 120 unidades de saúde (baixa e média complexidade - tipo I e II, respectivamente) e 1.669 crianças menores de 5 anos que procuraram os serviços públicos de saúde. O processamento e a análise dos dados foram realizados no Epi-info 6.02. Utilizouse o qui-quadrado de Pearson, com correção de Yates, para as tabelas binárias. A referida pesquisa foi aprovada pelo Comitê de Ética em Pesquisa da Universidade Federal de Pernambuco.

Tratando-se de uma avaliação complementar de dois estudos anteriores (I e II Pesquisa Estadual de Saúde e Nutrição - I e II $\mathrm{PESN}^{10,13}$ ), a pesquisa "Atenção à Saúde Materno-Infantil no Estado de Pernambuco" selecionou os 18 municípios anteriormente investigados em 1991 e 1998, respectivamen$\mathrm{te}^{10,13}$. Assim, da Região Metropolitana do Recife foram incluídos os municípios do: Recife, Cabo de Santo Agostinho, Jaboatão dos Guararapes, Olinda e Paulista; do Interior do Estado: Belém do São Francisco, Bodocó, Caruaru, Camocim de São Félix, Goiana, Itaíba, Itaquitinga, Orobó, Palmares, Panelas, Ribeirão, São Bento do Una e Triunfo. 
Para descrever as unidades de saúde e suas atividades, foram entrevistados $69 \mathrm{di}$ retores destes serviços na RMR e 51 do Interior, totalizando 120 unidades. Vale ressaltar que os responsáveis administrativos pelas unidades de saúde só foram informados de sua participação na composição da amostra na ocasião da coleta de dados, a fim de evitar possíveis implicações no funcionamento desses serviços que pudessem interferir nos resultados da avaliação.

Para obtenção dos dados foram utilizados dois formulários específicos direcionados às mães/acompanhantes das crianças após serem atendidas, para se avaliar o pro- cesso, e aos diretores das unidades de saúde, para avaliação de aspectos referentes à estrutura do serviço.

\section{Resultados}

Da amostra de crianças estudadas, $26,7 \%$ tinham menos de 6 meses, $21,4 \%$ entre 6 e 11 meses, $21,1 \%$ entre 12 e 23 meses, e $30,8 \%$ entre 24 e 59 meses, sem diferenças notáveis de distribuição etária entre a RMR e o Interior do Estado. Das 1.669 crianças, 116 não foram atendidas, o que resultou em uma amostra de 1.553 crianças. Em relação à escolaridade das mães, 9,4\% eram analfabetas

Tabela 1 - Características das crianças, mães e famílias atendidas nos serviços públicos do Estado de Pernambuco, 1998

Table 1 - Characteristics of children, mothers and families seen at public services in the State of Pernambuco, 1998

\begin{tabular}{|c|c|c|c|c|c|c|}
\hline \multirow[t]{2}{*}{ VARIÁVEIS } & \multicolumn{2}{|c|}{ RMR } & \multicolumn{2}{|c|}{ INTERIOR } & \multicolumn{2}{|c|}{ TOTAL } \\
\hline & $\mathrm{N}^{\circ}$ & (\%) & $\mathrm{N}^{\circ}$ & (\%) & $\mathrm{N}^{\circ}$ & (\%) \\
\hline \multicolumn{7}{|l|}{ CRIANÇAS } \\
\hline \multicolumn{7}{|l|}{ Sexo } \\
\hline Masculino & 459 & 51,9 & 401 & 51,1 & 860 & 51,5 \\
\hline Feminino & 425 & 48,1 & 384 & 48,9 & 809 & 48,5 \\
\hline \multicolumn{7}{|l|}{ Faixa etária - Idade (meses) } \\
\hline$<6$ & 253 & 28,6 & 192 & 24,5 & 445 & 26,7 \\
\hline $6-11$ & 194 & 21,9 & 163 & 20,8 & 357 & 21,4 \\
\hline $12-23$ & 182 & 20,6 & 171 & 21,8 & 353 & 21,1 \\
\hline $24-59$ & 255 & 28,8 & 259 & 33,0 & 514 & 30,8 \\
\hline Total & 884 & 53,0 & 785 & 47,0 & 1669 & 100,0 \\
\hline \multicolumn{7}{|l|}{ MÃES } \\
\hline \multicolumn{7}{|l|}{ Idade (anos) } \\
\hline $10-19$ & 170 & 19,3 & 129 & 16,5 & 299 & 18,0 \\
\hline $20-29$ & 532 & 60,5 & 460 & 59,0 & 992 & 59,8 \\
\hline$>30$ & 178 & 20,2 & 191 & 24,5 & 369 & 22,2 \\
\hline * Total & 880 & 53,0 & 780 & 47,0 & 1660 & 100,0 \\
\hline \multicolumn{7}{|l|}{ Grau de instrução } \\
\hline Analfabeta & 82 & 9,4 & 151 & 19,4 & 233 & 14,1 \\
\hline $\begin{array}{l}1^{\circ} \text { grau incompleto/ } \\
\text { completo }\end{array}$ & 594 & 68,0 & 513 & 65,9 & 1107 & 67,1 \\
\hline $\begin{array}{l}2^{\circ} \text { grau incompleto/ } \\
\text { completo }\end{array}$ & 188 & 21,5 & 106 & 13,6 & 294 & 17,8 \\
\hline Superior & 09 & 1,0 & 08 & 1,0 & 17 & 1,0 \\
\hline **Total & 873 & 52,9 & 778 & 47,1 & 1651 & 100,0 \\
\hline \multicolumn{7}{|l|}{ FAMÍLIA } \\
\hline \multicolumn{7}{|l|}{ Renda familiar $(S M)^{1}$} \\
\hline $0 \mathrm{a}<1$ & 275 & 31,1 & 385 & 49,0 & 660 & 39,5 \\
\hline $1 \mathrm{a}<2$ & 265 & 30,0 & 212 & 27,0 & 477 & 28,6 \\
\hline $2 a<3$ & 79 & 8,9 & 52 & 6,6 & 131 & 7,8 \\
\hline 3 e mais & 265 & 30,0 & 136 & 17,3 & 401 & 24,0 \\
\hline Total & 884 & 53,0 & 785 & 47,0 & 1669 & 100,0 \\
\hline
\end{tabular}

* 09 sem informação $/ 09$ without information; ** 18 sem informação /18 without information $\mathrm{SM}^{1}=$ Salário Mínimo/MW-Minium Wage; ${ }^{*} 01$ sem informação/1 without information 
na RMR e $19,4 \%$ no Interior, enquanto $2 / 3$ declararam ter cursado o $1^{\circ}$ grau incompleto/completo. No que se refere à renda familiar, $61,1 \%$ das crianças na RMR pertenciam a famílias que recebiam menos de dois salários mínimos por mês, proporção que se elevava para $76 \%$ na amostra do Interior (Tabela 1).

Na RMR, 97,1\% das unidades dispunham de cartão para registro de dados do crescimento, proporção que cai significativamente $(80,4 \%)$ no Interior $(\mathrm{p}<0,01)$. A existência de balanças pediátricas para a pesagem de menores de 2 anos foi notificada em quase 90,0\% das unidades de saúde da RMR e em $76,5 \%$ do Interior, situação bem aproximada dos valores encontrados em relação à existência de balanças para adultos, também usadas para crianças com dois ou mais anos. Apenas 20,3\% e 27,5\% dos serviços de saúde, respectivamente na RMR e no Interior, dispunham de normas para o acompanhamento do crescimento. Outros detalhes referentes aos aspectos estruturais acham-se descritos na Tabela 2.

Nos serviços de saúde estudados, $54,1 \%$ das crianças foram pesadas no dia da consulta e $16,2 \%$ medidas (comprimento em estatura). O registro do peso ao nascer constava na capa de $86,8 \%$ dos cartões, mas apenas em 36,9\% dos casos foi anotado no gráfico de acompanhamento. Quanto ao peso efetuado no dia da consulta, 53,0\% na RMR e $71,5 \%$ no Interior foram registrados no modelo gráfico de acompanhamento. No conjunto da amostra, apenas $18,2 \%$ das mães

Tabela 2 - Características da estrutura funcional (cartão da criança, balanças, normas e fichas) das unidades de saúde pesquisadas por espaços geográficos. Pernambuco, 1998

Table 2 - Functional structure characteristics (children's card, scales, standards and records) of the health units surveyed by geographical area. Pernambuco, 1998

\begin{tabular}{|c|c|c|c|c|c|c|c|}
\hline \multirow[t]{2}{*}{ CARACTERÍSTICAS } & \multicolumn{2}{|c|}{ RMR } & \multicolumn{2}{|c|}{ INTERIOR } & \multicolumn{2}{|c|}{ TOTAL } & \multirow[t]{2}{*}{ TESTE ESTATÍTICO } \\
\hline & $\mathrm{N}^{\circ}$ & (\%) & No & (\%) & No & (\%) & \\
\hline \multicolumn{8}{|l|}{ Existência de cartão da criança } \\
\hline Sim & 67 & $(97,1)$ & 41 & $(80,4)$ & 108 & $(90,0)$ & \multirow{3}{*}{$\begin{array}{l}\chi^{2} \text { Yates }=7,34 \\
p<0,01\end{array}$} \\
\hline Não & 02 & $(2,9)$ & 10 & $(19,6)$ & 12 & $(10,0)$ & \\
\hline Total & 69 & $(57,5)$ & 51 & $(42,5)$ & 120 & $(100,0)$ & \\
\hline \multicolumn{8}{|l|}{ Balança (até 2 anos) } \\
\hline Sim, em funcionamento & 62 & $(89,9)$ & 39 & $(76,5)$ & 101 & $(84,2)$ & \multirow{4}{*}{$\begin{array}{l}\chi^{2}=10,17 \\
p<0,01\end{array}$} \\
\hline Sim, com defeito & 06 & $(8,7)$ & 03 & $(5,9)$ & 09 & $(7,5)$ & \\
\hline Não & 01 & $(1,4)$ & 09 & $(17,6)$ & 10 & $(8,3)$ & \\
\hline Total & 69 & $(57,5)$ & 51 & $(42,5)$ & 120 & $(100,0)$ & \\
\hline \multicolumn{8}{|l|}{ Balança (>2 anos e adultos) } \\
\hline Sim, em funcionamento & 62 & $(89,9)$ & 45 & $(88,2)$ & 107 & $(89,2)$ & \multirow{4}{*}{$\begin{array}{l}\chi^{2}=0,34 \\
p=0,84\end{array}$} \\
\hline Sim, com defeito & 02 & $(2,9)$ & 01 & $(2,0)$ & 03 & $(2,5)$ & \\
\hline Não & 05 & $(7,2)$ & 05 & $(9,8)$ & 10 & $(8,3)$ & \\
\hline Total & 69 & $(57,5)$ & 51 & $(42,5)$ & 120 & $(100,0)$ & \\
\hline \multicolumn{8}{|c|}{ Normas p/acompanhamento do crescimento } \\
\hline Sim & 14 & $(20,3)$ & 14 & $(27,5)$ & 28 & $(23,3)$ & \multirow{4}{*}{$\begin{array}{l}\chi^{2}=0,97 \\
p=0,61\end{array}$} \\
\hline Não & 51 & $(73,9)$ & 35 & $(68,6)$ & 86 & $(71,7)$ & \\
\hline Não sabe & 04 & $(5,8)$ & 02 & $(3,9)$ & 06 & $(5,0)$ & \\
\hline Total & 69 & $(57,5)$ & 51 & $(42,5)$ & 120 & $(100,0)$ & \\
\hline \multicolumn{8}{|c|}{ Fichas $p /$ acompanhamento do crescimento $p /$ serem anexadas ao prontuário } \\
\hline Sim & 15 & $(24,6)$ & 03 & $(7,7)$ & 18 & $(18,0)$ & \multirow{3}{*}{$\begin{array}{l}\chi^{2} \text { Yates }=3,53 \\
p<0,06\end{array}$} \\
\hline Não & 46 & $(75,4)$ & 36 & $(92,3)$ & 82 & $(82,0)$ & \\
\hline *Total & 61 & $(61,0)$ & 39 & $(39,0)$ & 100 & $(100,0)$ & \\
\hline
\end{tabular}

*20 sem informação/*20 without information 
receberam informações sobre a situação de crescimento de seus filhos (Tabela 3 ).

A maior parte dos atendimentos efetuados nas unidades de saúde se faz através dos médicos, seguidos dos auxiliares, de outros profissionais e, por fim, de enfermeiros, como se observa na Tabela 4. No atendimento às crianças, na RMR os profissionais solicitaram mais o cartão da criança do que nos serviços do Interior. A diferença de desempenho é estatisticamente significativa entre os dois espaços geográficos, excetuando-se o caso dos auxiliares de enfermagem, que solicitam o cartão da criança em $83 \%$ dos atendimentos na RMR e no Interior do Esta- do. Chama-se a atenção que, nos postos de saúde do Interior, apenas $1 / 4$ dos médicos usam os cartões das crianças em suas consultas (Tabela 4).

A análise dos gráficos de crescimento de 1.194 cartões, segundo os detalhes descritos na Tabela 5, evidencia que $38,0 \%$ dos casos apresentavam nenhum ou apenas um ponto de registro de peso. A condição "nenhum ponto registrado" distribui-se de forma praticamente uniforme em todos os grupos etários. A freqüência modal de registro ocorreu no intervalo de 2 a 6 pontos (40,5\%), com predominância em crianças menores de 1 ano $(46,0 \%)$.

Tabela 3 - Assistência prestada às crianças $<5$ anos atendidas em serviços públicos de saúde por espaços geográficos. Pernambuco, 1998

Table 3 - Care given to children < 5 years old seen at public health services by geographical area. Pernambuco, 1998

\begin{tabular}{|c|c|c|c|c|c|c|c|}
\hline \multirow[t]{2}{*}{ VARIÁVEIS } & \multicolumn{2}{|c|}{ RMR } & \multicolumn{2}{|c|}{ INTERIOR } & \multicolumn{2}{|c|}{ TOTAL } & \multirow[t]{2}{*}{ TESTE ESTATÍTICO } \\
\hline & $\mathrm{N}^{\circ}$ & $(\%)$ & $\mathrm{N}^{\circ}$ & (\%) & No & (\%) & \\
\hline \multicolumn{8}{|c|}{ Criança pesada } \\
\hline $\operatorname{Sim}$ & 476 & $(58,4)$ & 364 & $(49,3)$ & 840 & $(54,1)$ & \multirow{3}{*}{$\begin{array}{l}\chi^{2} \text { Yates }=12,50 \\
p<0,01\end{array}$} \\
\hline Não & 339 & $(41,6)$ & 374 & $(50,7)$ & 713 & $(45,9)$ & \\
\hline Total & 815 & $(100,0)$ & 738 & $(100,0)$ & 1553 & $(100,0)$ & \\
\hline \multicolumn{8}{|c|}{ Criança medida } \\
\hline Sim & 154 & $(18,9)$ & 98 & $(13,3)$ & 252 & $(16,2)$ & \multirow{3}{*}{$\begin{array}{l}\chi^{2} \text { Yates }=8,58 \\
p<0,01\end{array}$} \\
\hline Não & 661 & $(81,1)$ & 640 & $(86,7)$ & 1301 & $(83,8)$ & \\
\hline Total & 815 & $(100,0)$ & 738 & $(100,0)$ & 1553 & $(100,0)$ & \\
\hline \multicolumn{8}{|c|}{ Registro do peso ao nascer na capa do cartão } \\
\hline Sim & 615 & $(87,5)$ & 420 & $(85,7)$ & 1035 & $(86,8)$ & \multirow{3}{*}{$\begin{array}{l}\chi^{2} \text { Yates }=0,64 \\
p=0,42\end{array}$} \\
\hline Não & 88 & $(12,5)$ & 70 & $(14,3)$ & 158 & $(13,2)$ & \\
\hline${ }^{*}$ Total & 703 & $(100,0)$ & 490 & $(100,0)$ & 1193 & $(100,0)$ & \\
\hline \multicolumn{8}{|c|}{ Registro do peso ao nascer no gráfico do cartão } \\
\hline Sim & 267 & $(38,0)$ & 173 & $(35,3)$ & 440 & $(36,9)$ & \multirow{3}{*}{$\begin{array}{l}\chi^{2} \text { Yates }=0,78 \\
p=0,37\end{array}$} \\
\hline Não & 436 & $(62,00$ & 317 & $(64,7)$ & 753 & $(63,1)$ & \\
\hline *Total & 703 & $(100,0)$ & 490 & $(100,0)$ & 1193 & $(100,0)$ & \\
\hline \multicolumn{8}{|c|}{ Registro do peso no dia da consulta no gráfico do cartão } \\
\hline Sim & 206 & $(53,0)$ & 168 & $(71,5)$ & 374 & $(59,9)$ & \multirow{3}{*}{$\begin{array}{l}\chi^{2} \text { Yates }=20,19 \\
p<0,001\end{array}$} \\
\hline Não & 183 & $(47,0)$ & 67 & $(28,5)$ & 250 & $(40,1)$ & \\
\hline **Total & 389 & $(100,0)$ & 235 & $(100,0)$ & 624 & $(100,0)$ & \\
\hline \multicolumn{8}{|c|}{ Recebeu informação sobre crescimento } \\
\hline Sim & 198 & $(24,3)$ & 85 & $(11,5)$ & 283 & $(18,2)$ & \multirow{3}{*}{$\begin{array}{l}\chi^{2} \text { Yates }=4 \\
p<0,001\end{array}$} \\
\hline Não & 617 & $(75,7)$ & 653 & $(88,5)$ & 1270 & $(81,8)$ & \\
\hline Total & 815 & $(100,0)$ & 738 & $(100,0)$ & 1553 & $(100,0)$ & \\
\hline
\end{tabular}

* Total de crianças que levaram o cartão para a consulta (01 sem informação)/* Total number of children that took card to appointment (01 without information)

** Total de crianças atendidas, pesadas no dia da consulta e possuíam o cartão no dia da entrevista/** Total number of children seen, weighed on the day of appointment and that had the card on the day of the interview 
Tabela 4 - Solicitação do cartão segundo profissional responsável pelo atendimento às crianças por espaços geográficos. Pernambuco, 1998

Table 4 - Card requesting according to professional responsible for child care by geographical areas. Pernambuco, 1998

\begin{tabular}{|c|c|c|c|c|c|c|c|}
\hline \multirow[t]{2}{*}{ SOLICITAÇÃO DO CARTÃO } & \multicolumn{2}{|c|}{$\mathrm{RMR}$} & \multicolumn{2}{|c|}{ INTERIOR } & \multicolumn{2}{|c|}{ TOTAL } & \multirow[t]{2}{*}{ TESTE ESTATÍTICO } \\
\hline & $\mathrm{N}^{\circ}$ & (\%) & No & (\%) & $\mathrm{N}^{\circ}$ & (\%) & \\
\hline \multicolumn{8}{|l|}{ Médico } \\
\hline Cartão solicitado & 340 & $(66,9)$ & 109 & $(24,7)$ & 449 & $(47,3)$ & \multirow{3}{*}{$\begin{array}{l}\chi^{2} \text { Yates }=167,06 \\
p<0,001\end{array}$} \\
\hline Cartão não solicitado & 168 & $(33,1)$ & 332 & $(75,3)$ & 500 & $(52,7)$ & \\
\hline Total (atendimentos) & 508 & $(100,0)$ & 441 & $(100,0)$ & 949 & $(100,0)$ & \\
\hline \multicolumn{8}{|l|}{ Enfermeiro } \\
\hline Cartão solicitado & 119 & $(90,8)$ & 07 & $(58,3)$ & 126 & $(88,1)$ & \multirow{3}{*}{$\begin{array}{l}\chi^{2} \text { Yates }=8,20 \\
p<0,01\end{array}$} \\
\hline Cartão não solicitado & 12 & $(9,2)$ & 05 & $(41,7)$ & 17 & $(11,9)$ & \\
\hline Total & 131 & $(100,0)$ & 12 & $(100,0)$ & 143 & $(100,0)$ & \\
\hline \multicolumn{8}{|l|}{ Auxiliar de enfermagem } \\
\hline Cartão solicitado & 269 & $(83,0)$ & 260 & $(83,3)$ & 529 & $(83,2)$ & \multirow{3}{*}{$\begin{array}{l}\chi^{2} \text { Yates }=0,00 \\
p=0,99\end{array}$} \\
\hline Cartão não solicitado & 55 & $(17,0)$ & 52 & $(16,7)$ & 107 & $(16,8)$ & \\
\hline Total & 324 & $(50,9)$ & 312 & $(49,1)$ & 636 & $(100,0)$ & \\
\hline \multicolumn{8}{|l|}{ Outro } \\
\hline Cartão solicitado & 68 & $(51,9)$ & 65 & $(36,9)$ & 133 & $(43,3)$ & \multirow{3}{*}{$\begin{array}{l}\chi^{2} \text { Yates }=6,26 \\
p<0,05\end{array}$} \\
\hline Cartão não solicitado & 63 & $(48,1)$ & 111 & $(63,1)$ & 174 & $(56,7)$ & \\
\hline Total & 131 & $(100,0)$ & 176 & $(100,0)$ & 307 & $(100,0)$ & \\
\hline
\end{tabular}

Tabela 5 - Número de pontos registrados no gráfico de crescimento (cartão) segundo a idade da criança em 120 serviços de saúde do Estado de Pernambuco, 1998

Table 5 - Number of dots recorded in the growth chart (card) according to child's age in 120 health services in the State of Pernambuco, 1998

\begin{tabular}{lrrrrrrrr}
\hline${ }^{*}$ NÚMERO DE PONTOS & \multicolumn{3}{c}{$<1$ ANO } & \multicolumn{2}{c}{$1-2$ ANOS } & \multicolumn{2}{c}{$3-5$ ANOS } & \multicolumn{2}{c}{ TOTAL } \\
\hline Nenhum ponto & 182 & $(27,8)$ & 87 & $(21,7)$ & 38 & $(27,5)$ & 307 & $(25,7)$ \\
01 ponto & 104 & $(15,9)$ & 31 & $(7,7)$ & 12 & $(8,7)$ & 147 & $(12,3)$ \\
2 a 6 pontos & 301 & $(46,0)$ & 141 & $(35,2)$ & 41 & $(29,7)$ & 483 & $(40,5)$ \\
07 a 11 pontos & 63 & $(9,6)$ & 80 & $(20,0)$ & 21 & $(15,2)$ & 164 & $(13,7)$ \\
$>12$ pontos & 05 & $(0,8)$ & 62 & $(15,5)$ & 26 & $(18,8)$ & 93 & $(7,8)$ \\
Total & 655 & $(100,0)$ & 401 & $(100,0)$ & 138 & $(100,0)$ & 1194 & $(100,0)$ \\
\hline
\end{tabular}

$\chi^{2}=143,50 \quad p<0,001$

\section{Discussão}

Embora o crescimento e o desenvolvimento sejam os dois suportes em torno dos quais devem gravitar as ações de promoção, proteção e recuperação da saúde das crianças, segundo enunciados doutrinários e normativos do Ministério da Saúde há mais de 20 anos, na realidade dos serviços assistenciais de Pernambuco esta recomendação não está sendo praticada, na grande maioria dos serviços e dos itens avaliados. Considerando-se o trinômio estrutural (pessoal, balanças/cartões e normas escritas) verifica-se que a restrição inicial para a vigilância do crescimento situa-se na questão de instruções normativas para acompanhar o processo, visto que apenas $23,3 \%$ das unidades de saúde referiam sua existência. Em resumo: dispõe-se de balanças pediátricas $(84,2 \%)$ ou de adultos $(89,2 \%)$, de cartão $(90,0 \%)$, de pessoal (médico, enfermeiro ou 
outro profissional), mas menos de $1 / 4$ dispõem ou pelo menos sabem da existência de normas e de fichas para padronizar a avaliação e suas implicações no âmbito dos cuidados e das ações demandados.

Esta observação facilita o entendimento das omissões e das falhas assinaladas no âmbito do desempenho ou do processo de vigilância do crescimento. Em outro estudo ${ }^{3}$, nos mesmos serviços de saúde, percebeu-se a confusão conceitual e operativa dos profissionais entrevistados sobre crescimento e desenvolvimento, e sobre quem, quando e como cuidar das demandas em relação aos dois aspectos, a partir da idéia simplista e hegemônica da separação já superada de puericultura e pediatria, e portanto entre crescimento e desenvolvimento e seus desvios.

Mesmo o conceito mais superficial derivado da prática dos serviços de saúde com os sucessivos programas que demandam o setor saúde, como os programas nutrição em saúde (1974), suplementação alimentar (1984), complementação alimentar (1990), combate as carências nutricionais (1995) e bolsa alimentação (2000), ainda assim a avaliação do crescimento não foi instrumentalizada na rotina dos serviços de saúde. É uma norma que, em suma, não é devidamente valorizada.

Isto explicaria, como se observa com os resultados aqui analisados, o fato de se dispor quase sistematicamente da informação do peso registrado na capa do cartão, mas o dado não é transferido para o gráfico de acompanhamento, da mesma forma que o peso obtido nas consultas convencionais, muitas vezes anotado no prontuário mas não representado no modelo gráfico da criança. Explicaria também o fato de que, só por exceção, as mães são informadas sobre o crescimento, perdendo-se assim o caráter instrutivo que a informação deve proporcionar.

O desinteresse pela monitoração do crescimento, comum nos serviços de saúde do Estado, é particularmente proeminente no Interior, de forma que os testes estatísticos, nas diversas comparações efetuadas, comprovam esta distribuição de comportamen- tos, com exceção apenas do registro do peso ao nascer no gráfico de crescimento, igualmente deficiente nas duas situações (RMR, com $38,0 \%$, e Interior, com $35,3 \%$ ). Não se dispõe, no banco de dados utilizado, de elementos analíticos para explicar esta diferenciação entre os dois espaços geográficos, podendo-se conjeturar que a maior demanda de usuários, a supervisão menos efetiva dos serviços e a própria condição das famílias (menor renda e níveis mais baixos de escolarização) estariam desfavorecendo as populações radicadas fora da área metropolitana do Estado.

No que se refere a esta discriminação de cuidados em relação ao processo de crescimento cabe referir, de modo específico, o comportamento dos médicos no Interior em relação ao item "cartão solicitado pelo responsável pelo atendimento”. Enquanto na RMR, $66,9 \%$ dos médicos adotam tal procedimento nas consultas, no Interior apenas $24,7 \%$ seguem esta orientação. É um fato muito ilustrativo, desde que o médico é, convencionalmente, o profissional hegemônico dos serviços de saúde, de forma que o seu aparente desinteresse pela monitoração do crescimento pode resultar ou induzir um efeito negativo na própria conduta dos outros profissionais.

Ademais, a propósito do possível desempenho dos profissionais, expresso no cuidado elementar de solicitar o cartão da criança no momento do atendimento, há de se ressaltar os registros positivos dos auxiliares de enfermagem, desde que $83,1 \%$ deles adotam este procedimento em suas intervenções. Esta singularidade, se por um lado distingue a ação dos auxiliares de enfermagem, por outro conduz a outros questionamentos. Além de solicitar o cartão, que registros, análises e decisões concretas tais profissionais realizam em relação ao item crescimento da criança? Que tipo de capacitação receberam, em serviços que, inclusive, não dispõem de normas a respeito do tema?

Não é surpreendente verificar que apenas $18 \%$ das mães/acompanhantes tenham recebido alguma informação sobre o crescimento de seus filhos no momento da con- 
sulta, sendo que, nos casos do Interior do Estado, apenas 11,5\% dos entrevistados relataram tal conduta. Os profissionais responsáveis pelo atendimento das crianças deveriam aproveitar o momento da consulta para orientar as mães e/ou acompanhantes, transmitindo-lhes conhecimentos de saúde e nutrição de seus filhos, no contexto de sua vida cotidiana $^{14,15}$.

Na realidade, a importância do crescimento da criança é um aspecto que transita por todos os objetivos e estratégias dos cuidados de saúde. Um simples episódio diarréico, mesmo constituindo uma patologia breve e autolimitada, com duração de três dias em mais de $90 \%$ dos casos, interfere até em processos de longa duração, como no crescimento esquelético, como demonstrou Bittencourt, em áreas faveladas do Rio de Janeiro ${ }^{16}$.

Vidal, Frias e Marques ${ }^{17}$, com o objetivo de avaliar o grau de implantação do Programa de Atenção Integral à Saúde da Criança (PAISC) em Pernambuco, constataram um baixo grau de implantação para o conjunto das ações, tendo como piores desempenhos o controle das infecções respiratórias agudas $(13,8 \%)$ e o crescimento e desenvolvimento $(10,8 \%)$.

É evidente que doenças de ação patogênica mais intensa e prolongada, como broncopneumonias, meningoencefalites, nefropatias, doenças metabólicas e, por sua escala epidemiológica, os desvios nutricionais, como as anemias, com freqüência de $40 \%$ a $90 \%$, a hipovitaminose $\mathrm{A}$, com a prevalência de 16 a $55 \%$ em crianças no Nordeste ${ }^{18}$ e a deficiência de iodo, ao lado de enteroparasitoses e de infecções bacterianas e viróticas, estabelecem um amplo espectro de comorbidades que podem comprometer o processo de crescimento. $\mathrm{O}$ aspecto nutricional ou, mais especificamente, a desnutrição energético-protéica, é apenas um elo nesta cadeia de eventos, que não pode ser omitida na assistência integral à saúde da criança.

Infelizmente, a experiência dos serviços públicos de saúde no Estado de Pernambuco demonstra haver ainda um longo caminho a ser trabalhado, no sentido de se implantar um processo efetivo e eficaz de vigilância ao crescimento de menores de cinco anos.

\section{Referências}

1. UNICEF. Declaração Mundial sobre a Sobrevivência, a Proteção e o Desenvolvimento da Criança. Nações Unidas, Nova York; 1990.

2. Figueira F, Ferreira OS, Alves JGB. Carta do IMIP. Pediatria, Rio de Janeiro: Medsi; 1996. p. 1-4.

3. Ratis CAS. Monitoramento do crescimento e desenvolvimento de crianças atendidas em serviços públicos de saúde do Estado de Pernambuco [dissertação de mestrado]. Recife: Departamento de Nutrição: área de concentração: Saúde Pública da Universidade Federal de Pernambuco; 2003.

4. UNICEF. Estratégia para melhorar a nutrição de crianças e mulheres nos países em desenvolvimento: um exame de políticas. Nova York; 1990.

5. OPAS/OMS. Ações de saúde materno-infantil a nível local, segundo as metas da Cúpula Mundial em favor da infância. Washington, DC; 1997.

6. Ministério da Saúde. Centro de Documentação do Ministério da Saúde. Textos básicos de saúde. Assistência Integral à Saúde da Criança: ações básicas. Brasília (DF); 1984.
7. Donabedian A. Explorations in quality assessment and monitoring: the methods and finding of quality assessment and monitoring. Michigan: Healthy Administration Press; 1985. p. 1771-81. v.3.

8. Batista Filho M. Alimentação, Nutrição e Saúde. In: Rouquayrol MZ \& Almeida Filho N. (Org.).

Epidemiologia \& Saúde. Rio de Janeiro: Medsi; 1999. p .353-74.

9. IBGE - Instituto Brasileiro de Geografia e Estatística. Pesquisa Nacional por amostra de domicílios - PNAD, 1999. Rio de Janeiro: IBGE; 2000.

10. Secretaria de Saúde do Estado de Pernambuco. II Pesquisa Estadual de Saúde e Nutrição: saúde, nutrição, alimentação e condições socioeconômicas no estado de Pernambuco. Recife: Governo do Estado de Pernambuco/ INAN/ MS-IMIP-DN/ UFPE; 1998.

11. Batista Filho M, Romani SAM. Alimentação, Nutrição e Saúde no Estado de Pernambuco. Recife: Instituto Materno Infantil de Pernambuco, IMIP; 2002. (Série Publicações Científicas do Instituto Materno Infantil de Pernambuco, IMIP, n.7). 
12. Ministério da Saúde. Atenção à Saúde MaternoInfantil no Estado de Pernambuco. Recife: MS/IMIP/ UFPE/DN/SES/PE; 2000.

13. Governo do Estado de Pernambuco/UNICEF. Crianças e adolescentes em Pernambuco: saúde, educação e trabalho. Brasília (DF): UNICEF; 1992.

14. Schall VT, Struchiner M. Educação em saúde: novas perspectivas. Cad Saúde Pública 1999; 15(2): 4-6.

15. Samico IC. Avaliação dos serviços no ambulatório geral de pediatria - IMIP. Revista do IMIP (Edição Especial) 1995; 9(2): 51-6.
16. Bittencourt SA, Leal MC, Santos MO. Hospitalizações por diarréia infecciosa no Estado do Rio de Janeiro. Cad Saúde Pública 2002; 18(3): 747-54.

17. Vidal SA, Frias PG de; Marques NM. Avaliação normativa das ações do Programa de Atenção Integral à Saúde da Criança (PAISC) em Pernambuco. Rev Br Saúde Materno-Infantil 2001; 1(2): 129-35.

18. Santos LMP. Distribuição espacial das carências de micronutrientes prevalentes no Brasil. Relatório ao Ministério da Saúde. Recife; 1999 (mimeo).

recebido em: 22/08/2003 versão final reapresentada em: 19/01/2004 aprovado em: 20/01/2004 